\title{
Two chitin synthase genes from Ustilago maydis
}

\author{
Beatriz Xoconostle-Cázares, ${ }^{1}$ Claudia León-Ramirez ${ }^{1}$ \\ and José Ruiz-Herrera ${ }^{1,2}$
}

Author for correspondence: José Ruiz-Herrera. Tel: +52462 51600. Fax: +52 46245849.

Departamentos de Genética y Biología Molecular ${ }^{1}$ and Ingeniería Genética (Unidad Irapuato) ${ }^{2}$, Centro de Investigación y de Estudios Avanzados del IPN, Irapuato, Gto, Mexico

\begin{abstract}
PCR was used to amplify fragments corresponding to CHS genes from Ustilago maydis, utilizing as primers oligonucleotides devised according to the conserved regions of fungal CHS genes. The PCR product was employed as a probe to screen a genomic library of the fungus. Two different CHS genes (Umchs1 and Umchs2) were thus identified in the positive clones recovered. Their sequence revealed high similarity with the CHS genes previously cloned from other fungi, especially in their central region. Alignment with the deduced protein sequences of all CHS genes reported up to date showed the existence of seven conserved domains. Transcripts from both genes were detected in the yeast and mycelial forms. In general, the transcripts from the Umchs1 gene appeared to be present at a higher level than the transcripts from the Umchs2 gene; the transcripts from both genes appeared to be more abundant in the mycelial form. Gene replacement of either gene and analysis of the resulting phenotype demonstrated that they are non-essential. Nevertheless, growth, chitin synthase activity levels, and chitin content of mycelial cells induced by cultivation in acidic media were all reduced in chs 1 and chs 2 mutants. However, mating, virulence and dimorphic behaviour were unaffected. Overall, the results indicate that the CHS1 and CHS2 genes encode products with redundant functions in $U$. maydis.
\end{abstract}

Keywords: Ustilago maydis, chitin synthases, cell wall, gene replacement

\section{INTRODUCTION}

Chitin is the most important structural component of the cell wall of fungi (Ruiz-Herrera, 1992). It is not surprising then that great effort has been dedicated to the study of the mechanisms involved in its synthesis. Results obtained with different fungi have demonstrated that chitin synthase, the enzyme responsible for the synthesis of the polymer, is accumulated in specific microvesicles, called chitosomes, which convey the enzyme from its site of synthesis to the cell surface, where chitin formation takes place (Bracker et al., 1976; Bartnicki-Garcia et al., 1978; Ruiz-Herrera, 1984). More data exist on the genetic control of chitin synthase than on the enzyme itself. By use of genetic and molecular biology approaches, it has been concluded that two types of chitin synthases exist in fungi: those encoded by $\mathrm{CHS}$ genes contain a single

Abbreviation: GICNAC, $\mathrm{N}$-acetylglucosamine.

The GenBank/EMBL/DDBJ accession numbers for the Umchs1 and Umchs2 sequences reported in this paper are $X 87748$ and $X 87749$, respectively. polypeptide chain and are activated by proteolysis in vitro; and those encoded by $C S D$ (or $C A L$ ) genes which contain more than one polypeptide, and are not believed to be activated by proteolysis in vitro (Bulawa, 1993). In all systems studied thus far, more than one CHS gene has been detected (Bowen et al., 1992; Bulawa, 1993). Gene disruption of one of these genes is not lethal in most fungi, suggesting that there is functional redundancy.

An attractive possibility for the multiplicity of genes encoding chitin synthase is that they are involved in wall synthesis at different stages during cell differentiation. Specific roles during the cell cycle of Saccharomyces cerevisiae have been suggested for the different chitin synthases (Shaw et al., 1991), although it has been demonstrated that chitin synthase III encoded by CSD2 (or CAL1) contributes to the synthesis of most of the chitin of the cell (Valdivieso et al., 1991; Bulawa, 1992). This enzyme also appears to be responsible for the formation of the chitosan layer during sporulation (Pammer et al., 1992). A study of the two CHS genes of Candida albicans demonstrated that CHS2 was differentially expressed during the dimorphic 
transition of the fungus (Chen-Wu et al., 1992). Nevertheless, disruption of this gene had no effect on its dimorphic behaviour (Gow et al., 1993).

Ustilago maydis is a plant pathogen responsible for corn smut. During its haploid saprophytic phase, the fungus grows in the form of budding yeast-like sporidia. When two compatible strains mate, they invade the plant and the dikaryotic phase grows in mycelial form. Mycelial growth of $U$. maydis can also be obtained transiently on solid medium during mating of compatible strains, or by forced diploids (reviewed by Banuett, 1992). The existence of two $C H S$ genes in the fungus was demonstrated by PCR amplification using as primers oligonucleotides designed for the more conserved region of $C H S$ genes from $S$. cerevisiae (Bowen et al., 1992). Gold \& Kronstad (1994) analysed the expression of both genes using these PCR fragments as probes, and carried out gene disruption. They concluded that they were equally expressed, and that neither gene was essential for mating or pathogenicity. We have recently described conditions which permit the dimorphic transition of haploid strains of the fungus in liquid or solid media (Ruiz-Herrera et al., 1995). We have now cloned two $C H S$ genes of $U$. maydis to study their expression during the dimorphic transition, and the phenotypic alterations brought about in mutants carrying substitutions of these genes.

\section{METHODS}

Strains and culture media. Strains FB1 $\left(a_{1} b_{1}\right)$ and FB2 $\left(a_{2} b_{2}\right)$ of $U$. maydis were obtained from Flora Banuett (University of California, San Francisco, USA). They were maintained and propagated as previously described (Ruiz-Herrera et al., 1995). Yeast growth in liquid media occurs at $\mathrm{pH} 7$, whereas mycelial growth is induced at $\mathrm{pH} 3$. Growth was measured as $\mathrm{OD}_{600}$ (Pharmacia Ultrospec III spectrophotometer) and calculated as protein by use of a calibration curve. Fuzz reaction on solid charcoal-containing medium was performed as described by Holliday (1974). Escherichia coli strains JM103 and KW258 were used for transformation, and for plasmid and phage propagation, respectively; they were grown in LB medium. For plasmid propagation, the bacteria were grown in TB medium containing the necessary antibiotics for selection. For phage propagation, LB medium was supplemented with $10 \mathrm{mM}$ $\mathrm{MgSO}_{4}$ and $2 \%(\mathrm{w} / \mathrm{v}$ ) maltose.

PCR amplifications. Oligonucleotides corresponding to the conserved regions of $C H S$ genes from $S$. cerevisiae, designed by Bowen et al. (1992) were synthesized, and used as primers for PCR. Their sequences are as follows: oligo 5', 5' CTG AAG CTT AC(TGCA) ATG TA(TC) AA(TC) GA(GA) GAC 3', and oligo $3^{\prime}, 5^{\prime}$ GTT CTC GAG (TC)TT (GA)TA (TC)TC (GA)AA (GA)TT (TC)TG $3^{\prime}$. The following conditions were employed: 30 cycles; melting step, $1 \mathrm{~min}$ at $94^{\circ} \mathrm{C}$; oligonucleotide annealing, $1 \mathrm{~min}$ at $50^{\circ} \mathrm{C}$; polymerization, $2 \mathrm{~min}$ at $72^{\circ} \mathrm{C}$. The bands obtained were ligated to PCRII vector (Invitrogen) and transformed into E. coli JM103.

Transformation of $\boldsymbol{U}$. maydis. The procedure described by Sanchez et al. (1993) for Kluyveromyces lactis was slightly modified. Cells were harvested by centrifugation after reaching $\mathrm{OD}_{600} 1.5$ and resuspended in fresh culture medium containing $10 \mathrm{mM}$ DTT. After $30 \mathrm{~min}$ cells were recovered by centrifugation, washed four times with deionized water and resuspended in water to a final concentration of $10^{10}$ cells $\mathrm{ml}^{-1}$. Cells $(40 \mu \mathrm{l})$ were electroporated with a Bio-Rad electroporator in $0.2 \mathrm{~cm}$ cuvettes under the following conditions: $1500 \mathrm{~V}, 25 \mathrm{mF}$ and $400 \Omega$. Efficiency of transformation under these conditions using an autonomous replication vector was of the order of $8 \times 10^{5}-1 \times 10^{6}$ transformants $\left(\mu \mathrm{g}\right.$ DNA) ${ }^{-1}$

DNA techniques. Restriction endonucleases and modifying enzymes were obtained from BRL and used according to the supplier's recommendations. Oligonucleotides were synthesized using a DNA synthesizer (Applied Biosystems) by reversed-phase chromatography. Southern's techniques were carried out as described by Maniatis et al. (1982).

Hybridization. Double-stranded DNA probes were labelled with $\left[\alpha_{-}{ }^{32} \mathrm{P}\right] \mathrm{dCTP}$ by random priming according to the manufacturer's instructions (USB). Nylon membranes containing DNA samples were hybridized under high stringency conditions with the radiolabelled probe. Washing was carried out at $60^{\circ} \mathrm{C}$ with $2 \times$ SSC (Maniatis et al., 1982).

Screening of the genomic library. The genomic library of $U$. maydis RK32 $\left(a_{2} b_{3}\right)$ constructed in $\lambda$ EMBL- 3 was obtained from Regine Kahmann (Institute für Genetik und Mikrobiologie, Universität München, Germany). The screening was carried out using as a probe the PCR product labelled by random priming. Positive clones were amplified in $E$. coli KW258. To identify by restriction analysis the phages containing sequences related to CHS, DNA was isolated, digested with Bam HI, EcoRI and SalI, and probed by Southern hybridization with the radiolabelled PCR product. Fragments detected with the probe were subcloned in pBluescript for further analysis.

DNA sequencing. Sequencing of DNA was performed by the dideoxynucleotide chain-termination method of Sanger et al. (1977) using the Sequenase kit (version 2.0; USB). Single- or double-stranded DNA were used as templates. The terminal sequences of the cloned fragments were obtained using the forward and reverse universal primers. Additional primers were designed to extend these sequences and also to resolve ambiguous regions.

RNA extraction and Northern blotting. $U$. maydis yeast and mycelial cells were grown according to the method of RuizHerrera et al. (1995) for $24 \mathrm{~h}$. Cells were collected by centrifugation and washed with diethyl-pyrocarbonate-treated water. The pelleted cells were ground with a mortar, cooling with liquid nitrogen. Total RNA was isolated using the acid guanidine thiocyanate procedure (Chomczynski \& Sacchi, 1987). RNA was separated in formaldehyde/agarose gels, transferred to Hybond-N + membranes (Amersham) and hybridized with the radiolabelled probe under stringent conditions (Maniatis et al., 1982).

Strategy for gene replacement. A vector for $U m c h s 1$ gene replacement was constructed as follows: a Bam HI fragment of Umchs $11.7 \mathrm{kbp}$ in length was subcloned in a pBluescript $\mathrm{KS}(-)$ vector, from which the $S_{a} / \mathrm{I}$ site had been previously deleted. A HindIII linker was cloned in the NruI site of the insert. Then the HindIII-SalI fragment of Umchs1 (30 codons in length) was removed and substituted by a $2.6 \mathrm{kbp}$ HindIII-SalI fragment containing the hygromycin-resistance cassette from pCM54 (Tsukuda et al., 1988). For Umchs2, the strategy was as follows: a $1.5 \mathrm{kbp} B a m \mathrm{HI}$ fragment from Umcbs2 was cloned into pBluescript $\mathrm{KS}(-)$ from which the $X b o \mathrm{I}$ site had been previously deleted. A HindIII linker was cloned in the NruI site of the insert. The internal HindIII-XboI fragment, which contains 76 codons, was substituted by the $2.6 \mathrm{kbp}$ HindIII-SalI hygromycin-resistance cassette.

Additional methods. Cell-free extracts were obtained with a Braun homogenizer or by grinding in a mortar with liquid 
GTGCTTGATTCTTGGTTGATCGGCCUACGCUACATGGC TATTA TCGAGACGGTT CGGCCGGAGG CGCGCACAGGACGATCMGACCCATATCC

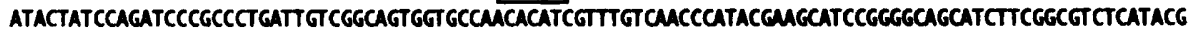
TCTCCCTTCTCGGATGAGCATGCTGCATCTCCTCGCCGGCATCCATTCTGCCCCTCTCGCATCAGCAGTGTCTGCTCATGCACGCAGCAGCMCACATG M S M L H L L A G I H S A P L A S A S V C S C T Q Q Q H M

AGCATCAGCGGGATCCGGGGATGGGAGCAGTTCGCATGCCTGGGCTCAGCGGAGTTTACAGCUGCAGCGATGGCTCGCTACCCCACCGCCC

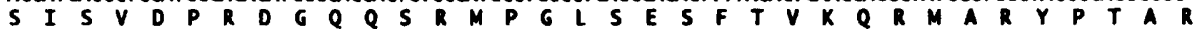
GCGCAGGCGCTTIMGCCCATCAGCTCACCTTGCCACGCTACCCGAGCACTCCCAGGCTCCCCTGTCAGGCTCCGTCGATGGAGATATCATCTACAGCC A G A L S P S A H L A T L PE H S Q A P L S G S V D G E Y HL Q P AGAGCGCCGCTGGACACTACTCATCGCTAGCT CACCGCCATTGGCOAGaCGATGMGACGACGACGTGCCGAGACMATACTGCCTAGCTCGGCGCAT E R R T T L L I A S S P P L A K T M K T T TM P R Q I L P S S A H

GATGMUATATGCATATGATCGTCGGATTCAGCGGCAGCCGGUCTTCTCCCTTTGGMGAGCAGCTGGTATCGCGTACTTGCAGAGCCCATATGCTCA D E K Y A $Y$ D R P I Q R Q P E L L P LEE Q L V S R T C R A H M L K AGTTGCACGCUACGATGACGACGACGAGGATGAGGCGCCGAGGATCCATACCGGCTTGACGCGTGTTCTGCTTTCGGAGGCGACAACGGCCAMGGCTA $L$ H A T M T T T R M R R R R S I P V L T R D S A F G G D NG Q G $Y$ TGACCCAMATTCTGCCTATGGTGGCGCCGGTATGGCTGGACTACCGGTCAGTTTGGCGTMCCACTTTGACACGCAACACTTTGGTCCCGCTCCACGA D P N S A Y G G A G Y G T T G Q F G D N H F D T Q H F G PA P R

CGCGGGCTCAGCTTCGTAGGCACMGACCUCU CUCGTACGCCTCACHUCUACCTCATTCTGGATTGCCCGTGCCEACCMGTTGCAGACGTTCC R G A Q L R R H K T K K N V R L T K K P TCACGCGACGAGCCGAGACGATTCACCACGATGCGTTATTCGGCCGTCACCTGCGATCCGACGGATTTGGAGCGATCCTTCACGCTTCGACCAGCGCT $T R R A E D E F T T M R Y S A V T C D P T D L E R S F T I R P A L$ GACGGTGACACACGGACTCTTCATCGCCATCACCATGACHCGMGACGAGTGCTITCTGTCGACTTTCCACGGCGTGATCAMUACATTGCG Y G R H T E L F I A I T M Y N

CACCTCTGTTCCCGCACAAGTCACGCACATGGGGCMMGATGGCTGGUMUGTGGGGTCGCCATCATCTCGGACGGGCGCAMGMGATTCACCCTA H L C S R N K S R T W G K D G W K K V V V A I I S D G R K K I H P R GAGGCTCGACTGCCTCGCCGCACTAGGAGTTACCUGACGGAGTGGCGMUACATGGTCGTGGCMUGMGTACGTGCGCATCTTTACCMUTACAC $V L D C L A A L G V Y Q D G V A K N M V D G K E V R A H L Y E Y T$

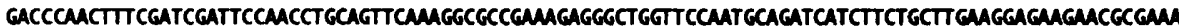
T Q L S I I D S N L Q F K G A E R G L V P M Q I I F C L K E K N A K AAGATCAATTCGCATCGATGGTCTTCMCGCGTTCTGCCCTATCCTGCUCCCUACGTCACCATCTTGTGGAGGTAGGACACGACCCGGACACAGT X I N S H R F F N A F C P I L Q P N V T I L L E V G T R P G D K S CGATTTACTACCTGTGGMATCGTTGATG GMCTCCUCGTTGCCGCGCATGTGGAGAGATCTGCGCCGAGACCAMGGCAMTGGGGAGGGGACC I Y Y L W K S F D N S V A A C G E I C A E T K G K W G G P

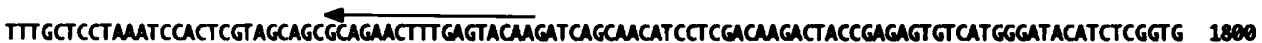
$L L L N P L V A$ A Q N F E Y K I S I L D K T T E S V M G Y I S V

CTTCCCGGTGCATTCTCCGCTTACCGCTACATCGCGCTGCAGUCGACGAATTGGCCATGGACCTCTGGCTTCCTACTTCMUGGCGUMACTTGTGG 1900 L P G A F S A Y R Y I A L Q N D E F G H G PLA A S Y F K G E NLL L GACGTGACGCTGACGTTTCACATCCACATGTACCTCGCCGMGATCCATCTTGGCTTCGGTTGGCGCCGUACGTGGGCATGGATGGGGTTGM 2600 R D A D V F T S N M Y L A E D R I L C F E L A A K R G H G W V L K GTACGT CMATCAGCTCG GGAGT GACCGATTTCCCGGGGACTCCCCGMTTCATCAGTCACCGACG CGTTGTTAMCGGATCCTCACCCACGTT 21EO Y V K S A R G V T D V P E G L P E F I S Q R R R W L N G S S P T S

TTGGAGACCCGACTTCAACTCCGCGGCATTGGCGTATTCMTGGTTATGCAGTACATCTATCTCGGTACTGGGTATCGGCTTCATCTTCGCGA 2200

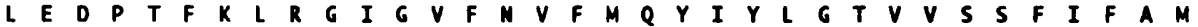
TGGGTAACCACCGCAGGGMGCMGTGGMGTACTGGGCTGCCGTCGCGGITGCTCTGCTIACCGTGACATGATGGTCGCCGCCGTCTGTGIT Z3CO G N R P Q G S K W Y A A V V V F A L L T V Y M M V A A V L C L

GAGCMGGTCGTCGTCGAGTAGACACGATGCCATCTATGCTCAGATGGTGGTCGCTTCTGGCCACGTACGCAGGTATCTGATCTCGTCGCTTCTC 24EO S K V V A R V E H D A I Y A Q M V V S L L A T Y G V Y L I S S L L GCGTCCGTCCTITGCACCTGATCACGTCTTCCTCCAGTACCTTCTGCTTGCACCGACCTACATCACATCCTCACATCTACAGCCTTCTGTMCTGG 2500

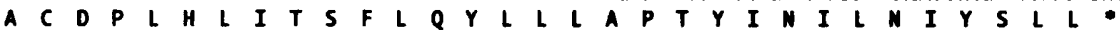

CACGATATGTCCTGGGGTACGAGGGGAGGCCGATGCACATCTCCGTGACCATGGACTTCGATAGGCTCCMAGGGCCATTCCMACTGCCTCTGG 2600 ACGCTAСCATTCCGCTCCCGCTGG GATGACGCCTCCCTTGTTTCTGGGTACTACTCCTGGGTCTCACCGTITTACATGACCCGCTCCAAGGA 2700 GATCCCTGGCTTGGGMTGTCGGTGGCACTAGCGG CGGATCTCTGTATTTMTGMGGGGGCAGATTMGMACGATMACACGTCGACAT 2800 MTMRTCTITCATCTTATATT

103 200 400 500 600 700 800 99 1000 1100 1200 1300 1400 1560 1600 1700 1800 1000
100
100
200
200

Fig. 1. DNA and deduced amino acid sequence of Umchs1. The ORF ( $2280 \mathrm{nt}$ ) is shown with the predicted amino acid sequence ( 760 amino acids). The putative TATA and polyadenylation signal sequences are boxed. Oligonucleotides used to generate the PCR probe are indicated by arrows.

nitrogen. No significant difference in chitin synthase activity was observed when either method was used. Cell walls were recovered by centrifugation at $2000 \mathrm{~g}$, washed succesively with
$2 \mathrm{M} \mathrm{NaCl}$ and water, and freeze-dried. The initial supernatant was centrifuged at $165000 \mathrm{~g}$ (Rav), and the pellet (mixed membrane fraction) was used as source of chitin synthase. Chitin 


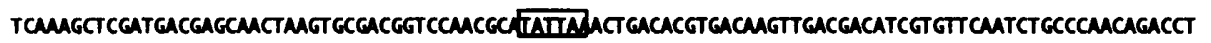

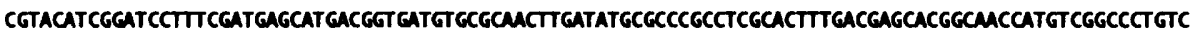
$M R P P$ R T L T S T A T M S A L S

GACMGCATGCCGATGGAGATTGACGTG GATGGACGMGACGCT TCMCGGCCAGGCGAGAGGCCGACTGCTCCACACGCMATTCCCCACGATGAC T S M P M E I D D V M D E D A V N G Q A R G R L L H T Q V P H D D

GATTACATGCCCGGTGGCTTCGATCCCACGTGCTCGMUATGGCCAGGCTGGAGGTTGCGCTTCGGCMGATCCCACHACGGTGCCMGGACGATACA D Y M P G G F D P N V L E N G Q A G G V R F G K I P Q R V P R R Y K

AGACGCTCAGAGGGT GAACTTACCATGGCMCCTCGCCTCGATTGCCCGTCCCCTCOMGCTGCTTGACMGCTCMACGACCAGAGTCCCGAG T L K R V L Y H G L V L D C P V P S L L L K L N D R E S R E ATTCACCCATATGCGATACACTGCTGCCACTTGCGACCCCGCGGTTCMUACCGAGCGTTACACATTGCGACAGGTTCTCTTTGACCCTCCTCGTCGC F T H M R Y T A A T C D P D E F K T E R Y T L R Q V L F D P P R R

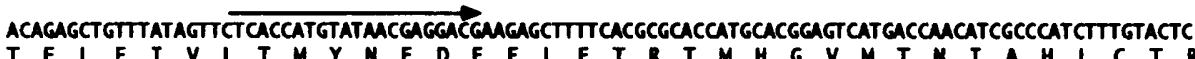
G R T M H G V M T N I A H L C T GAGAGCGCTCCMAACCTGGGGCUGCUGGCTGGMGMUGT CGT CGTCT TCATCGTCTCGGACGGCCGTCTCMGATCMCTCGCGCACCTTGGCGTG E R S K T G K E G K K V V V C I V S D G R L K I N S R T LA C CCTTGCTGCGTGGGAGTTACCAGGAGGGCGGGGGCAGMCGTGTCMTGGCAMCCCGTCACTGCTCACATCTACCUTACACAGCTCAGCTCTCG $L A A M G V Y Q E G G G$ G N V V G K P V T A H I Y E Y T A Q L S

ATCGATCCTAGCATGCACTTCAAGGGTGCOMGGCATCATGCCCGTCCMATTCTCTTCTGCCTCMGGAGCGCHCCACMGMGGATCAACTCGCACC I D P S M H F K G R E G I M P V Q I L F C L K E R N Q K K I N S H R GTTGTTCTTCMCGCTTITGGCCAGATCTTGCAGCCCMCATCTGCGTACTGCTTGATGAGGMCCATGCOAGACCTCGCTCCATTTACCATCTCTG W F F A F Q I L Q P N C V L L D V G T M PRPRSI Y H L W GMAGCCTITGACATCUCTCCHCGTCGCGGTTCCTGGGTGGATTGTCGCACTCMGGGCMUTTITGGGGGGCACTGCTCMTCCACTCGTCGCG K A F D I N S N V A G S C G E I V A L K G K F W G L L N P L V A GCGCMUATTCGAGTACMGATGTCCMCATTCTCGACMGCCGCTGGAGTCGGCTTTGGCTACATCACGGGCTTCCAGG GCCTTCTCCGCGTACC A Q N F E Y K M S N I L D K P L E S V F G Y I T V L P G A F S A Y R GCTACATTGCGTACAMACGAGCTCACGGTCMGGGCCCTTGGCTCGTACTTTMGGGTGUACATTACACGGGGCCAGTCGGCGCAGACGTCT Y I A L Q N D A HG Q G P L S Y F K G T L HGGQSDA D V F TACGTCGACATGATCTTGCCGAGGATCGCATCTTGGTTGGGGCTAGTGCGUGCGAGACAGCGCCTGGATCTTGCATTATGTCAGTTGCCCM T S N M Y L A E D R I L C WE L V S K R D S A W I L H Y V K S A Q

GCAGTCACGGATGGCCCCACCAAGTTCCCCACTGATCTCGCACGCCGTCGTTGGCTTMCCAGGATCCATTCGCGGCGCTCTACGCCCANTTCACT A V T D V P D Q V P E L I S Q R R R W L N Q D P F A A L Y A Q L H F TCTACMGGTCTGGACCAGCMGGCACTCGTTCGGCCGMAGATATTCCTCCAGGTCGATTCCTCTACCAGTTCATACMCTGCTATTCTCCTGGTTCTC Y K V TSK HSFGRKIFLQVEFLYQFIQLLFS F S

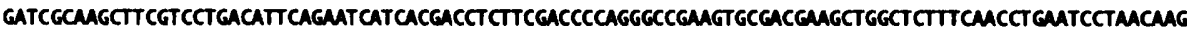
I A S F V L T F R I I T T S S T P G P K C D E A G S F N L N P K GAGACTTCTTCGCTCTTCTCGAGCGCTACGAGACATTGATCCCATCMTCGCCGTTGCGTCATCTTGGAGTTGTCATGCTCCTCMGGCGCAM E T S S L L L E R L R D I D P I NRR C V Y L G S L S M L L K A $Q S$ GCAMGACGCTCGAGCTGCAGCCTCTTCTCAGGCCGGGCCGTCTCGTCGGCCTCGACATT GACTCGGCCGGCCNGTTGTTCGATTGGTATGGGAGAG K T L E L Q P L L R P G R L V S A S T L TRPAKLF V L V WES CGAGACCCAGCGTCMCGGTGCCGCCCAGMGCTCGTCATCTCCTGGTTTGACGCCTGCGGAGGCGACCTTGTTATTCCTCACCCATTCGACGTGG E N P A S T V P P R S S S S P G L T PAEA T L L Y S S T N S T W TCGCTTCCHGCACATCGUGCAGCACTTCTCAGCATCTTGGAGACGCGTCGGCTCTCGTGCTCAGGTCTCTTCGATTCCGACCAMTTCMGTCGCC $S$ L P S T S R Q H F S A S W R V R L S S L R S R S I P T N S S R P

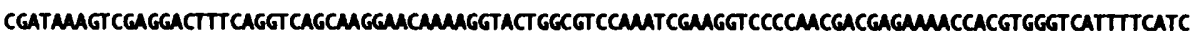

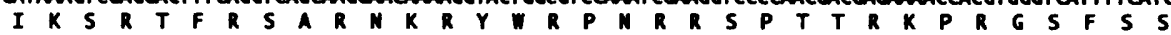
TTGGTCGCACT GGTCATGGGMACTCTCACCTTCATGCCTGGTGCGTCTCGCCCGCTCCATAGGCTCGATCTACCTCATGAGACCGCGTMCGAC W Q L V N G L S P S C P G L L P A P

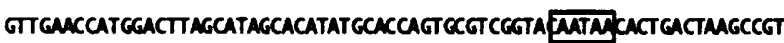

Fig. 2. DNA and deduced amino acid sequence of Umchs2. The ORF ( $2214 \mathrm{nt})$ is shown with the predicted amino acid sequence (738 amino acids). The putative TATA and polyadenylation signal sequences are boxed. Oligonucleotides to generate the PCR probe are indicated by arrows.

synthase activity was measured by the filtration method originally described by Ruiz-Herrera \& Bartnicki-Garcia (1976)

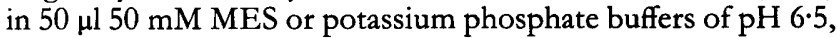
containing $0.25 \mathrm{M}$ sucrose, $10 \mathrm{mM} \mathrm{MgCl}$ or $\mathrm{MnCl}_{2}, 40 \mathrm{mM} \mathrm{N}$ acetylglucosamine (GlcNAc), $0.5 \mathrm{mM} \mathrm{UDP}\left[{ }^{14} \mathrm{C}\right] \mathrm{GlcNAc}$ (sp. act. $\left.18 \mathrm{mCi} \mathrm{mmol}^{-1} / 6.66 \times 10^{8} \mathrm{~Bq} \mathrm{mmol}^{-1}\right)$, and where indicated
$40 \mu \mathrm{g}$ trypsin $\mathrm{ml}^{-1}$. Activity was expressed as nmol GlcNAc incorporated by $1 \mathrm{mg}$ protein in $1 \mathrm{~min}$. For chitin measurement, cell walls were hydrolysed with $6 \mathrm{M} \mathrm{HCl}$, and glucosamine was measured by the Elson-Morgan method as described by Dische (1962), after $\mathrm{HCl}$ evaporation at room temperature under vacuum. Glucosamine content was related to wall neutral sugars 


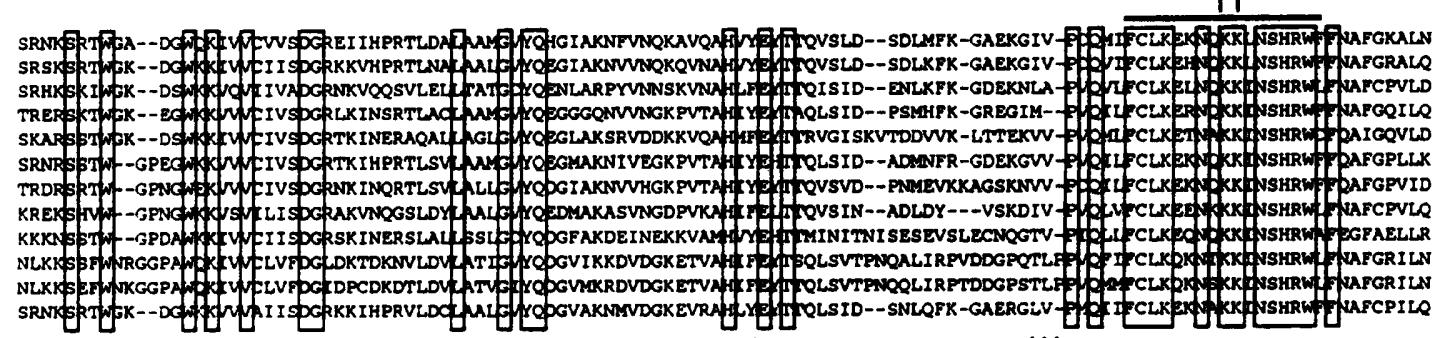

\author{
III
}
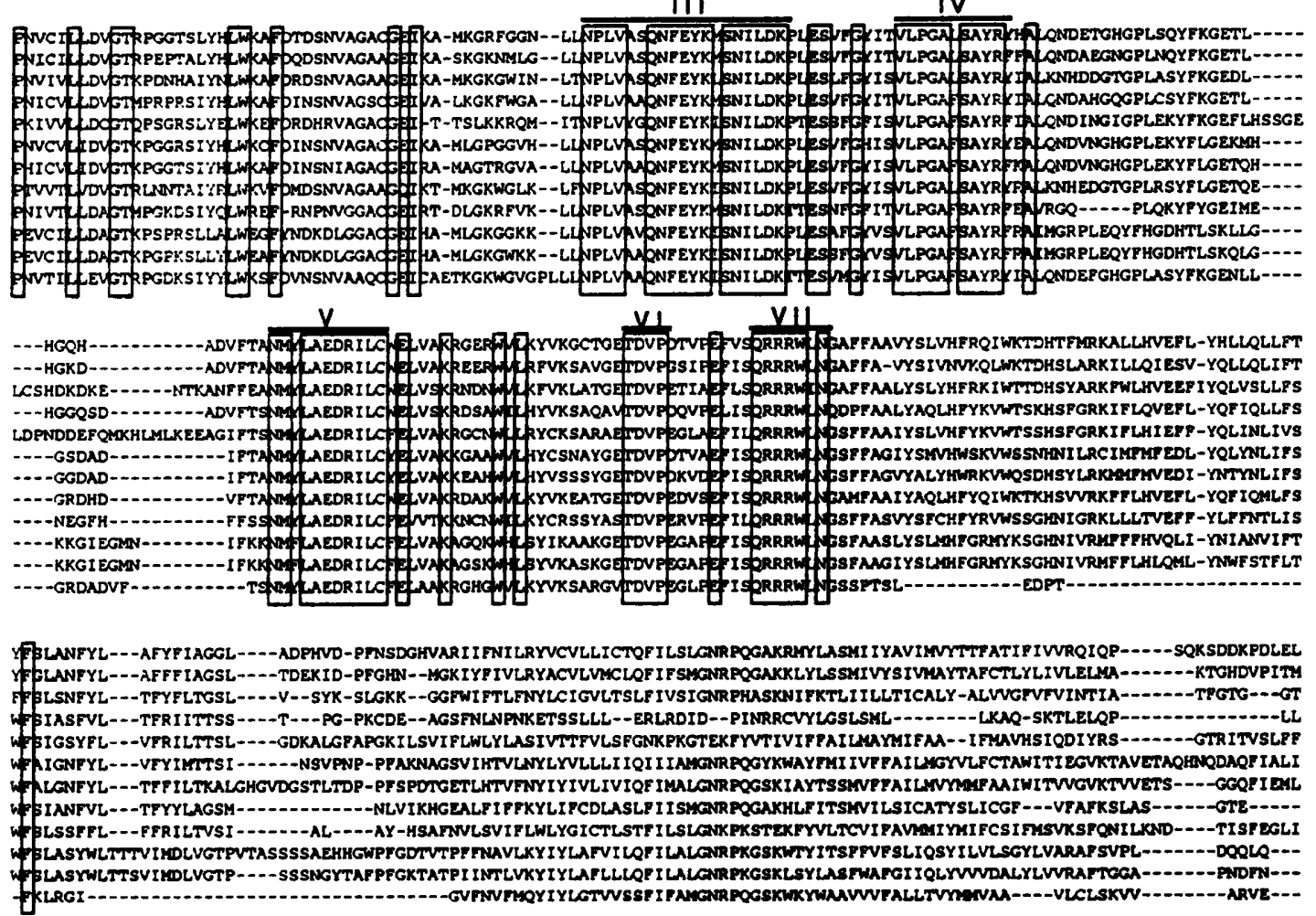

Fig. 3. Multiple amino acid sequence alignment of CHS genes deposited in GenBank as derived by maximal homology with GeneWorks software. Comparison of the sequences is indicated in single-letter code. Only regions displaying homology are shown. Boxes indicate complete identity at that position among all sequences, gaps are indicated by hyphens. Roman numerals indicate conserved domains (see Table 1). Abbreviations: NcCHS1, $N$. crassa CHS1; NcCHS2, $N$. crassa CHS2; AnCHSA, A. nidulans CHSA; AnCHSB, A. nidulans CHSB; ScCHS1, S. cerevisiae CHS1; ScCHS2, S. cerevisiae CHS2; CaCHS1, C. albicans CHS1; CaCHS2, C. albicans CHS2; RoCHS1, R. oligosporus CHS1; RoCHS2, R. oligosporus CHS2; UmCHS1, U. maydis CHS1; UmCHS2, U. maydis CHS2.

measured in $2 \mathrm{M} \mathrm{HCl}$ hydrolysates with anthrone (Dimler et al., 1952), to have an internal standard to compare the samples.

For the determination of virulence, 10 -d-old corn plantlets were injected with $0.2 \mathrm{ml}$ of a suspension of a mixture of $U$. maydiscompatible mating strains $\left(10^{8}\right.$ cells $\mathrm{ml}^{-1}$; five plants per mixture). Plants were incubated in a greenhouse and observed at intervals for the appearence of the galls, characteristic of the disease, which normally occurred after $10-20 \mathrm{~d}$.

\section{RESULTS}

\section{Isolation of PCR products}

Using the procedure and primers described in Methods, a major band of about $600 \mathrm{bp}$ was obtained and cloned. Restriction analysis of different clones containing the fragment showed three different restriction patterns, 
suggesting that they corresponded to more than one PCR product. This was confirmed by Southern analysis of $U$. maydis DNA digested with different restriction enzymes probed with one of the PCR fragments; in all cases two bands were observed. Sequencing of the two putative different PCR products showed ORFs whose predicted amino acid sequences displayed high similarity with both CHS1 and CHS2 gene products of S. cerevisiae (not shown), but displayed differences with those reported by Bowen et al. (1992) for U. maydis (see below).

\section{Cloning and sequencing of two CHS genes}

Using one of the inserts as a probe, we screened a genomic library of the fungus. Twenty positive signals were obtained out of 40000 recombinant phages screened. The phages were isolated, amplified, and their DNA was isolated, digested with different restriction enzymes and subjected to Southern analysis. Phages 7 and 12 were selected as representatives of the two different $C H S$ genes, and their restriction fragments were subcloned for further analysis.

\section{Umchs1 gene}

The structural gene and the regulatory sequences were contained in plasmids pi6, p7-1 and pii6. Fig. 1 shows the Umchs1 nucleotide and amino acid deduced sequences. The sequence shows a single continuous ORF, encoding a polypeptide of 760 amino acids and a molecular mass of $85106 \mathrm{Da}$. The predicted amino acid sequence of Umchs 1 is 26 and $30 \%$ similar to S. cerevisiae CHS1 and CHS2, respectively. No consensus splicing sequences occur in this gene. The non-translated $5^{\prime}$ and $3^{\prime}$ regions were analysed for consensus regulatory sequences. A putative TATA box is present at position 43. A consensus polyadenylation signal was also identified.

\section{Umchs2 gene}

The Umcbs2 gene was contained in p12, pb5 and pa7 plasmids. The complete DNA and deduced amino acid sequence appears in Fig. 2. A continuous ORF of 738 amino acids defining a polypeptide with a theoretical molecular mass of $83520 \mathrm{Da}$ is shown. The predicted amino acid sequence of $U m c b s 2$ is 27 and $35 \%$ similar to S. cerevisiae CHS1 and CHS2, respectively. In the $5^{\prime}$ nontranslated region a putative TATA box is present, whereas a polyadenylation signal at the $3^{\prime}$ end was identified.

\section{Sequence comparison of reported chitin synthase genes}

As indicated above, we noticed differences in the sequence of the PCR products synthesized by Bowen et al. (1992) and the corresponding fragments of $U m c h s$ genes reported here. At the amino acid level, similarities are of the following order: chs1 PCR product vs Umchs $1,58 \%$; and chs2 PCR product vs $U m c h s 2,61 \%$.
Table 1. Conserved domains in chitin synthase gene products

\begin{tabular}{|ll|}
\hline Domain* & \multicolumn{1}{c|}{ Sequence } \\
\hline I & T(M/Y)YNED \\
II & FCLK(Q/E)XNXKK(I/L)NSHRW \\
III & NPLV(A/Y)XQNFEYKXSNILDK \\
IV & VLPGA(F/L)SAYR \\
V & NM(F/Y)LAEDRILC \\
VI & TDVP \\
VII & QRRRW(L/I)N(G/Q) \\
\hline
\end{tabular}

* Domains are numbered starting at the amino terminus.

Using GeneWorks software (Intelligenetics, release 2.3) we made a comparison of the deduced amino acid sequences of $U$. maydis genes with those previously reported (Fig. 3). The amino acid sequences of the Umchs 1 and $U m c h s 2$ gene products show homology with products of the following genes: Rhizopus oligosporus CHS (unpublished results from T. Motoyama, GenBank accession nos D10159 and D10160); C. albicans CHS1 (Au-Young \& Robbins, 1990); C. albicans CHS2 (Chen-Wu et al., 1992); S. cerevisiae CHS1 (Bulawa et al., 1986), S. cerevisiae CHS2 (Silverman et al., 1988); Neurospora crassa CHS1 (Yarden \& Yanofsky, 1991), N. crassa CHS2 (Beth Din \& Yarden, 1994); and Aspergillus nidulans $C b s A$ and $C b s B$ (Yanai et al., 1994). This similarity is located in the central region of the genes, whereas the amino and carboxy termini showed only isolated conserved amino acids (not shown). Both proteins appear to be hydrophobic, mainly towards their carboxy termini. Analysis of possible modification motifs revealed several putative phosphorylation sites in both Umchs1 and Umchs2; and two putatives sequons in Umchs1 (data not shown).

According with the homology displayed, we have identified seven domains comprising at least four amino acids in all the sequences (Table 1). At least part of domain III and whole of domain VII are shared by CSD2 genes that have been cloned (Ruiz-Herrera \& Xoconostle-Cázares, 1995). With the sequence data a dendrogram of grouping of fungal chitin synthases was constructed (Fig. 4).

\section{Expression of Umchs1 and Umchs2 genes during dimorphism}

We determined the abundance of Umchs 1 and Umchs2 transcripts as a measure of the expression of both genes. Accordingly, Northern blot hybridization of total RNA obtained from the yeast or mycelial phases of the fungus was performed. Two non-homologous fragments, a 232 bp BamHI-XhoI fragment, and a 263 bp Bam HI-XhoI fragment, located towards the $3^{\prime}$ region of Umcbs 1 and Umchs2, respectively (see Fig. 6), were used as specific probes. A BglII fragment corresponding to the actin gene of Trichoderma reesei was used as a control. Our data (Fig. 5) indicate that $U m c h s 1$ transcripts are more abundant than Umchs2, and that the transcript levels from both 


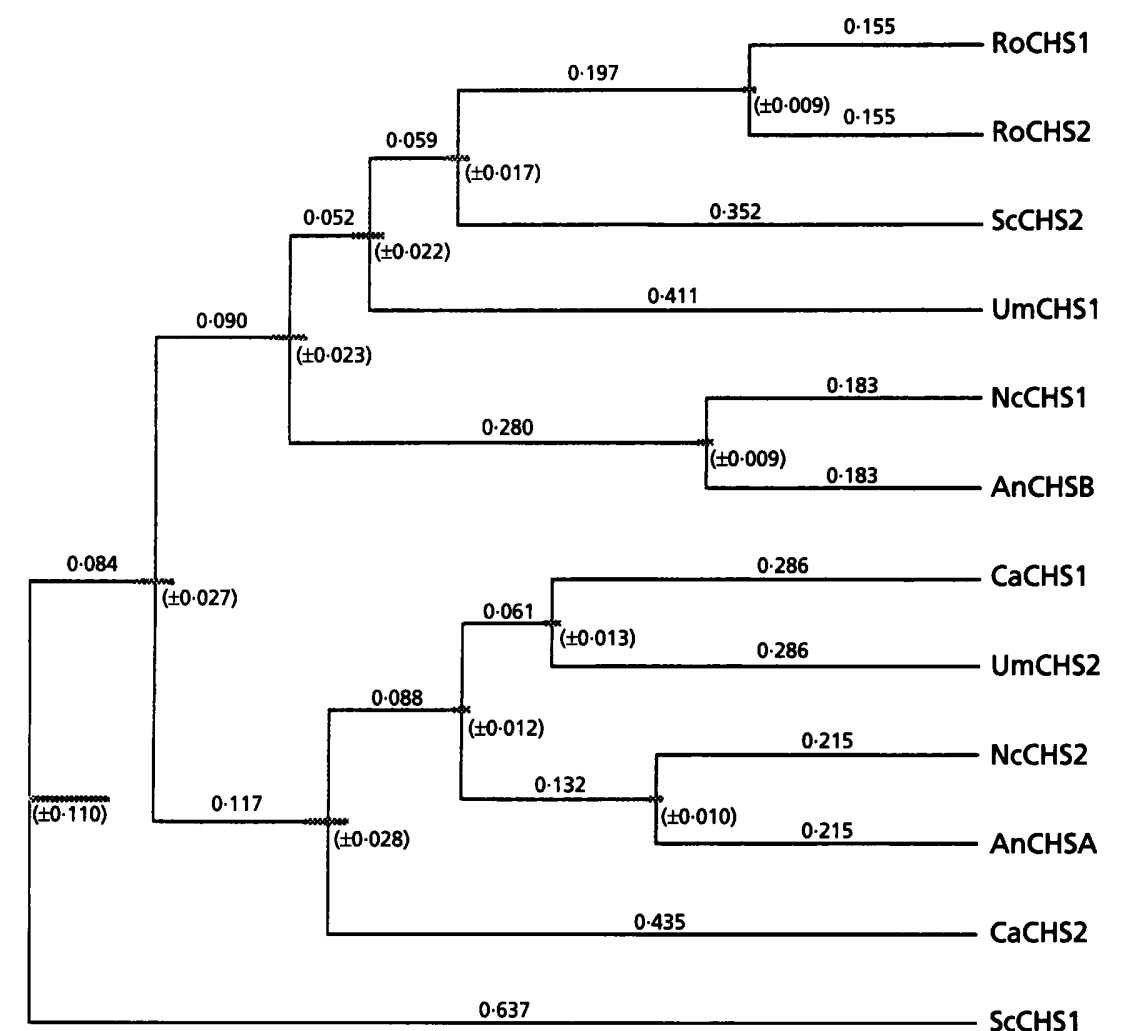

Fig. 4. Maximal parsimony dendrogram showing fungal chitin synthase grouping. The tree was calculated with GeneWorks software. Numbers in the branches are related to the differences between groups; numbers in parentheses show the SEM in the branching. Abbreviations are as in the legend to Fig. 3.

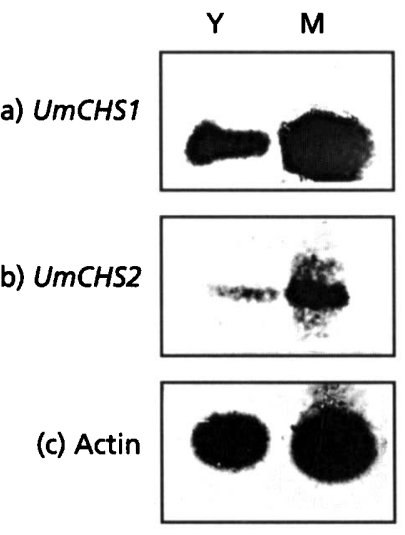

Fig. 5. Northern blot analysis. Total RNA was isolated from 24 h-old yeast or mycelium forms. (a) Blot was probed with the 232 bp BamHI-Xhol fragment of Umchs1. (b) Blot probed with the $263 \mathrm{bp} \mathrm{BamHI-Xhol} \mathrm{fragment} \mathrm{of} \mathrm{Umchs2.} \mathrm{(c)} \mathrm{Blot} \mathrm{was} \mathrm{re-}$ probed with the $T$. reesei actin gene. $Y$, Yeast RNA; $M$, mycelium RNA.

genes (but especially $U m c h s 2$ ), are higher in the mycelial stage of $U$. maydis.

\section{Gene replacement of Umchs genes}

The method for construction of the vectors for gene replacement is shown in Fig. 6. Cell transformation with the linear Bam HI fragments containing the replacement cassettes was performed by electroporation as described in Methods. Transformants were recovered in complete medium containing $1 \mathrm{M}$ sorbitol and $400 \mu \mathrm{g}$ hygromycin $\mathrm{B} \mathrm{ml}{ }^{-1}$. Gene replacement for both $c h s 1$ and $c h s 2$ mutants was confirmed by Southern analysis. Fig. 7 shows the results for FB2 strains. Identical results were obtained with FB1 (not shown).

\section{Phenotypic analysis of interrupted mutants}

In synthetic medium of $\mathrm{pH} 3$, which induces mycelial development, growth of both mutants was reduced as compared to wild-type (Table 2). Addition of $1 \mathrm{M}$ sorbitol did not restore normal growth (not shown). At $\mathrm{pH} 7$, growth was not affected. Cultivation in complete medium did not restore normal growth at $\mathrm{pH} \mathrm{3.0.} \mathrm{All} \mathrm{mutants}$ inoculated in synthetic or complex media of $\mathrm{pH} 7$ grew as normal yeasts; whereas in synthetic or complex medium of $\mathrm{pH} 3$ they grew in mycelial form (Ruiz-Herrera et al., 1995). All chs mutants displayed Fuzz colonial phenotype on charcoal-containing synthetic medium of $\mathrm{pH} 3 \cdot 0$.

Gene replacement had no effect on mating or virulence. Mating, as determined by the Fuzz reaction, was normal when the following pairs of strains (one $a_{1} b_{1}$, the other $\left.a_{2} b_{2}\right)$ were mixed: $c b s 1 \times$ wild-type; $c b s 1 \times c h s 1 ; c b s 1 \times c b s 2$; $c h s 2 \times$ wild-type; and $c h s 2 \times c h s 2$. All these mixtures were able to induce tumour formation in corn plants.

Determination of chitin synthase activity of wild-type and chs mutants grown in the yeast form at $\mathrm{pH} 7$, or in the mycelial stage at $\mathrm{pH} 3$ was performed as described in Methods. Two metal cations, $\mathrm{Mg}^{2+}$ or $\mathrm{Mn}^{2+}$, and two buffers, MES or phosphate, were used. Higher activity was obtained with MES buffer in the presence of $\mathrm{Mg}^{2+}$, 
UmCHS1

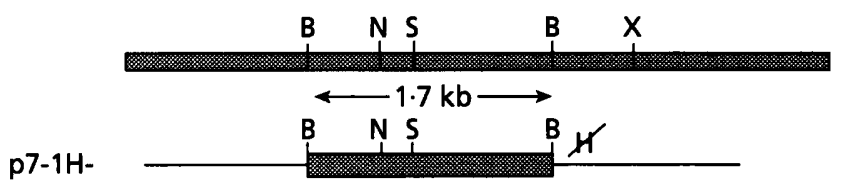

p7-15-
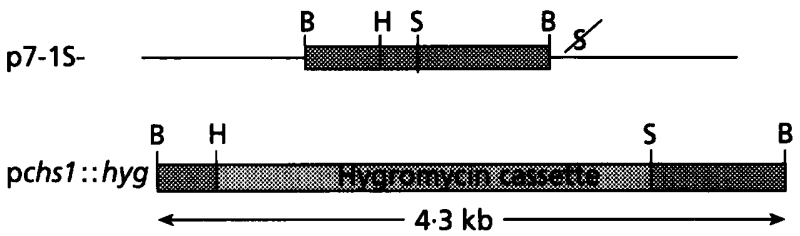

UmCHS2

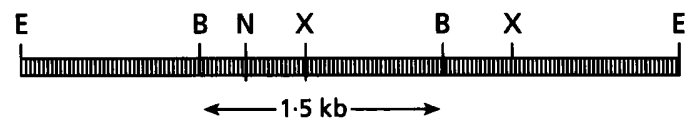

p12-2H-

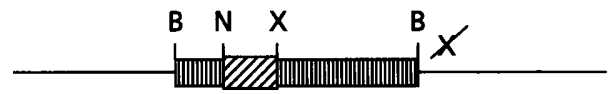

p12-2X-

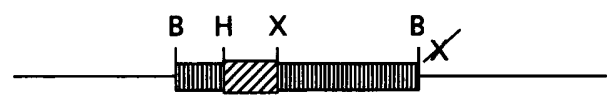

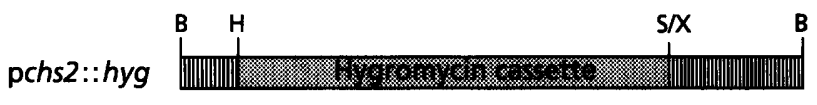

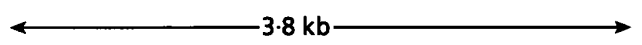

Fig. 6. Strategy for Umchs genes replacement. An internal conserved Umchs fragment was replaced by the hygromycinresistance gene. For details see text. Thin line is the pBluescript $\mathrm{KS}(-)$ vector. Restriction enzyme abbreviations: $\mathrm{B}, \mathrm{BamHI} ; \mathrm{H}$, HindIII; N, Nrul, S, Sall; X, Xhol.

but only when extracts from the yeast forms were tested; activity of mycelial extracts was very low (not shown). When phosphate and $\mathrm{Mg}^{2+}$ were employed, activity in yeast or mycelial extracts was rather similar. Activity in the $c h s$ mutants was lower than wild-type, but only when the extracts from the mycelial stage were analysed in the absence of trypsin; activity of the yeast cells with or without trypsin was about equal (Table 3). Addition of trypsin to the reaction mixtures decreased activity. This result contrasts with that for most fungi (Ruiz-Herrera, 1992).

Chitin levels in the cell wall were reduced in the yeast and mycelial forms of $c h s 2$ mutants, and slightly reduced in the mycelial form of $c h s 1$ strains (Table 4). (a)

(b)

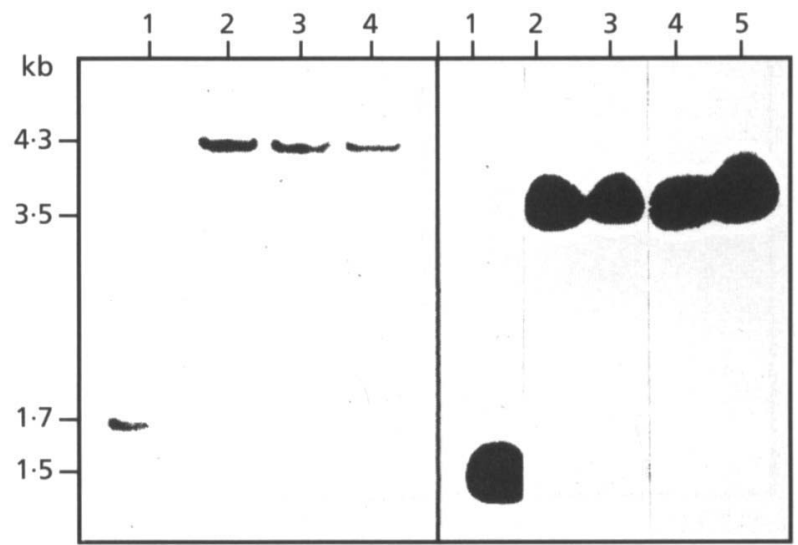

Fig. 7. Southern blot hybridization of chs mutants. (a) chs1 mutants. Lane 1, DNA from wild-type strain FB2; lanes 2-4, DNA from mutants. (b) chs2 mutants. Lane 1, DNA from wildtype strain FB2; lanes 2-5, DNA from mutants. DNA was digested with BamHI, electrophoresed and blotted onto a nylon membrane. Blot was probed with the fragment of $1.7 \mathrm{~kb} \mathrm{BamHI}$ from p7-1 for Umchs1, and with the $1.5 \mathrm{~kb}$ fragment from p12-2 for Umchs2.

Table 2. Comparison of growth of chs1 and chs2 mutants with wild-type in synthetic and complete medium

Inoculum was prepared in hygromycin-free medium. Cells were washed four times in water and inoculated into fresh medium as described in Methods to obtain yeast or mycelial growth in media of $\mathrm{pH} 7.0$ or 3.0 , respectively. Inoculum was of the order 15-20 $\mu \mathrm{g}$ protein $\mathrm{ml}^{-1}$ for all strains. Data are expressed as the ratio of growth between each mutant and wild-type, and are means \pm SEM of four experiments.

\begin{tabular}{|c|c|c|c|c|}
\hline \multirow[t]{2}{*}{ Medium } & \multirow[t]{2}{*}{$\mathrm{pH}$} & \multirow[t]{2}{*}{ Time (h) } & \multicolumn{2}{|c|}{ Growth ratio } \\
\hline & & & FB2chs1 & FB2chs2 \\
\hline \multirow[t]{6}{*}{ Synthetic } & $7 \cdot 0$ & 18 & $1.02 \pm 0.30$ & $1 \cdot 21 \pm 0 \cdot 09$ \\
\hline & & 24 & $1.01 \pm 0.06$ & $0.90 \pm 0.09$ \\
\hline & & 48 & $1.06 \pm 0.06$ & $1 \cdot 05 \pm 0.06$ \\
\hline & $3 \cdot 0$ & 18 & $1 \cdot 02 \pm 0 \cdot 10$ & $0.96 \pm 0.06$ \\
\hline & & 24 & $0.66 \pm 0.08$ & $0.68 \pm 0.08$ \\
\hline & & 48 & $0.54 \pm 0 \cdot 04$ & $0 \cdot 69 \pm 0 \cdot 10$ \\
\hline \multirow[t]{6}{*}{ Complete } & $7 \cdot 0$ & 18 & $2 \cdot 19 \pm 0 \cdot 50$ & $0.95 \pm 0.03$ \\
\hline & & 24 & $1 \cdot 10 \pm 0 \cdot 08$ & $0.95 \pm 0.02$ \\
\hline & & 48 & $1.00 \pm 0.06$ & $1.05 \pm 0.03$ \\
\hline & 3.0 & 18 & $0.96 \pm 0.05$ & $1 \cdot 18 \pm 0 \cdot 05$ \\
\hline & & 24 & $0.55 \pm 0 \cdot 13$ & $0.48 \pm 0 \cdot 10$ \\
\hline & & 48 & $0 \cdot 60 \pm 0 \cdot 03$ & $0.66 \pm 0.04$ \\
\hline
\end{tabular}

\section{DISCUSSION}

We have cloned and characterized two chs genes from $U$. maydis, which by their analogy to $S$. cerevisiae genes we have designated Umcbs 1 and Umchs2. They probably 
Table 3. Chitin synthase activity levels in $U$. maydis wildtype and chs mutants

Mixed membrane fraction was obtained from cells grown in complete medium at $\mathrm{pH} 7.0$ (yeasts) or $\mathrm{pH} 3.0$ (mycelium). Chitin synthase activity was measured as described in Methods in phosphate buffer in the presence of $\mathrm{Mg}^{2+}$. Data are expressed as nmol GlcNAc incorporated min $^{-1}$ (mg protein) ${ }^{-1}$. Data are means \pm SEM of two determinations from three different extracts.

\begin{tabular}{|lllcl|}
\hline \multirow{2}{*}{ Stage } & Trypsin & \multicolumn{3}{c|}{ Specific activity } \\
\cline { 3 - 5 } & & FB2 & CHS1 & CHS2 \\
\hline \multirow{2}{*}{ Yeast } & No & $21 \cdot 1 \pm 2 \cdot 6$ & $20 \cdot 7 \pm 1 \cdot 5$ & $20 \cdot 3 \pm 0 \cdot 8$ \\
& Yes & $11 \cdot 9 \pm 1 \cdot 4$ & $14 \cdot 2 \pm 1 \cdot 2$ & $13 \cdot 6 \pm 1 \cdot 7$ \\
Mycelium & No & $21 \cdot 6 \pm 3 \cdot 2$ & $12 \cdot 9 \pm 1 \cdot 3$ & $14 \cdot 2 \pm 1 \cdot 0$ \\
& Yes & $12 \cdot 6 \pm 1 \cdot 3$ & $11 \cdot 0 \pm 1 \cdot 3$ & $13 \cdot 0 \pm 1 \cdot 0$ \\
\hline
\end{tabular}

Table 4. Relative content of hexosamines and neutral sugars in the cell walls of $U$. maydis wild-type and chs mutants

Sugars were measured in walls obtained from yeast or mycelial cells obtained as described in Methods. Data are means \pm SEM of four different batches of cell walls.

\begin{tabular}{|c|c|c|}
\hline \multirow[t]{2}{*}{ Strain } & \multicolumn{2}{|c|}{ Ratio hexosamines/neutral sugars } \\
\hline & Yeast & Mycelium \\
\hline FB1 & $0 \cdot 19 \pm 0.011$ & $0.29 \pm 0.028$ \\
\hline FB1 cbs 1 & $0 \cdot 18 \pm 0 \cdot 017$ & $0.19 \pm 0.020$ \\
\hline FB1 chs 2 & $0 \cdot 13 \pm 0 \cdot 022$ & $0 \cdot 14 \pm 0.008$ \\
\hline FB2 & $0 \cdot 23 \pm 0 \cdot 020$ & $0.21 \pm 0.002$ \\
\hline FB2 chs 1 & $0 \cdot 23 \pm 0.023$ & $0.19 \pm 0.003$ \\
\hline $\mathrm{FB} 2$ chs 2 & $0 \cdot 13 \pm 0 \cdot 013$ & $0 \cdot 15 \pm 0 \cdot 015$ \\
\hline
\end{tabular}

correspond to the PCR fragments previously isolated by Bowen et al. (1992) from this fungus, and designated $c h s 1$ and $c h s 2$ by Gold \& Kronstad (1994), who used them for gene disruption. The differences observed in the sequence of the PCR products cbtained by those authors and the whole genes is probably due to the different strains employed. Further work (unpublished) has demonstrated the existence of a CSD2 gene in U. maydis. Accordingly, $U$. maydis follows the rule of other fungi, $S$. cerevisiae (Bulawa et al., 1986; Silverman et al., 1988; Bulawa, 1992, 1993; Valdivieso et al., 1991) and C. albicans (Au-Young \& Robbins, 1990; Chen-Wu et al., 1992; Sudoh et al., 1993), where the presence of both kinds of chitin synthase genes has been reported.

Comparison of the deduced amino acid sequences corresponding to both $C H S$ genes of $U$. maydis, with those cloned from other sources revealed that they have significant homologies in the central part of the molecules where seven highly conserved domains were identified. However, the amino and carboxy termini of $\mathrm{CHS}$ genes have no homology at all. It may be anticipated that the central region of the protein contains the domains corresponding to the active site of the enzyme, and possibly other regulatory domains. Our data on the conserved domains and the comparison dendrogram add further information to data described by Bowen $e t$ al. (1992) and Beth Din \& Yarden (1994). The most important difference between the analysis made with short PCR products (Bowen et al., 1992) and with the whole protein sequence (this study) is the disappearance of the originally described three $C H S$ classes, and the early divergence of two branches. As described here, CHS1 from $S$. cerevisiae appears as the least homologous CHS protein. Homology of many $C H S$ gene products does not appear to coincide with the accepted phylogenetic relationships of the fungi analysed. This inconsistency is difficult to explain unless ancient evolution of $C H S$ genes and convergence are invoked. It is interesting to note the high number of putative phosphorylation sites existing in both enzymes, mainly CHS1 product. Whether they are involved in regulation of enzymic activity, remains to be determined. As described above, $C H S$ gene products are activated in vitro by proteolysis (Bulawa, 1993).

Both genes were expressed in the yeast or mycelial forms of the fungus. We found a higher level of transcript for Umchs1, as compared to Umchs 2 in both mycelial and yeast-like stages, and increased levels of both transcripts in the mycelial phase, mainly for Umchs2. Gold \& Kronstad (1994) did not observe significant differences in the expression of either gene between haploids and mating cells which grow in filamentous form. This is probably due to the different protocols employed for mycelial growth induction, and the fact that we used haploid cells.

The observation that $U m c h s 1$ and $U m c b s 2$ are not essential agrees with the earlier report from Gold \& Kronstad (1994), who interrupted either gene with the corresponding PCR product. Our results on the null effect of $c h s$ gene substitution on mating and virulence also agree with the data obtained by those authors. These results are similar to the observation that both CHS1 and CHS2 from $S$. cerevisiae are dispensable genes (Bulawa et al., 1986; Bulawa \& Osmond, 1990). The levels of transcripts of Umchs1 and Umchs 2 were different in the yeast or mycelial forms of $U$. maydis; similar (although more pronounced) differences were reported for C. albicans (Chen-Wu et al., 1992). But as reported in this human pathogen (Gow et al., 1993), gene replacement in $U$. maydis did not affect the dimorphic transition induced by incubation at acid $\mathrm{pH}$. All these data suggest that either the function of both genes is interchangeable, or that a different chitin synthase, possibly the one encoded by the CSD2-like gene, is responsible for the synthesis of most of the chitin present in the cell wall, as is the case in S. cerevisiae (Valdivieso et al., 1991; Bulawa, 1992).

Although neither gene is essential, their substitution brought about some minor changes in the physiology of the null mutants, especially in the case of mycelial cells. Substitution of either Umchs1 or Umchs 2 provoked a 
reduction in growth rate of both mutants at $\mathrm{pH} 3$. Growth alteration does not appear to be due to an additional auxotrophy, since it was observed in synthetic or complex media. Besides, growth at $\mathrm{pH} 7$ in synthetic medium was almost unaffected and different isolates behaved similarly. In the case of $S$. cerevisiae, distuption of $C H S 1$ did not affect cell growth or morphology, but growth alterations in synthetic media were recorded (Bulawa, 1993). $U$. maydis mutants carrying either gene substitution displayed a reduction in the levels of chitin synthase activity in mycelial cells, as compared to wild-type. An additional change in chs 2 mutants was a reduction in the chitin content of the cell wall of both mycelium and yeast cells. Although minor, these changes suggest that some of the roles played by either gene product in cell wall construction can not be completely compensated by the nonaffected gene products.

\section{ACKNOWLEDGEMENTS}

This work was partially supported by CONACyT, México. B.X.-C. is a doctoral fellow from CONACyT. We thank Dr Regine Kahmann from the Institute für Genetik und Mikrobiologie der Universität München, Germany, for providing the genomic library of $U$. maydis in $\lambda E M B L-3$, and Dr Flora Banuett from the University of California, San Francisco, USA, for providing strains $\mathrm{FB} 1$ and $\mathrm{FB} 2$ of $U$. maydis.

\section{REFERENCES}

Au-Young, J. \& Robbins, P. W. (1990). Isolation of a chitin synthase gene (CHS1) from Candida albicans by expression in Saccharomyces cerevisiae. Mol Microbiol 4, 197-207.

Banuett, F. (1992). Ustilago maydis, the delightful blight. Trends Genet 8, 174-180.

Bartnicki-Garcia, S., Bracker, C. E., Reyes, E. \& Ruiz-Herrera, J. (1978). Isolation of chitosomes from taxonomically diverse fungi and synthesis of chitin microfibrils in vitro. Exp Mycol 2, 173-192.

Beth Din, A. \& Yarden, O. (1994). The Neurospora crassa cbs-2 gene encodes a non-essential chitin synthase. Microbiology 140, 2189-2197.

Bowen, A. R., Chen-Wu, J. L., Momany, M., Young, R., Szaniszlo, P. J. \& Robbins, P. W. (1992). Classification of fungal chitin synthases. Proc Natl Acad Sci US A 89, 519-523.

Bracker, C. E., Ruiz-Herrera, J. \& Bartnicki-Garcia, S. (1976). Structure and transformation of chitin synthetase particles (chitosomes) during microfibril synthesis in vitro. Proc Natl Acad Sci US $A$ 73, 4570-4574.

Bulawa, C. E. (1992). CSD2, CSD3, and CSD4 genes required for chitin synthesis in Saccharomyces cerevisiae: the CSD2 gene product is related to chitin synthases and to developmentally regulated proteins in Rhizobium species and Xenopus laevis. Mol Cell Biol 12, 1764-1776.

Bulawa, C. E. (1993). Genetics and molecular biology of chitin synthesis in fungi. Annu Rev Microbiol 47, 505-534.

Bulawa, C. E. \& Osmond, B. C. (1990). Chitin synthase I and synthase II are not required for chitin synthesis in vivo in Saccharomyces cerevisiae. Proc Natl Acad Sci US A 87, 7424-7428.

Bulawa, C. E., Slater, M., Cabib, E., Au-Young, J. \& Sburlati, A. (1986). The $S$. cerevisiae structural gene for chitin synthase is not required for chitin synthesis in vivo. Cell 46, 2113-2125.

Chen-Wu, J. L., Zwicker, J., Bowen, A. R. \& Robbins, P. W. (1992).
Expression of chitin synthase genes during yeast and hyphal growth phases of Candida albicans. Mol Microbiol 6, 497-502.

Chomczynski, P. \& Sacchi, N. (1987). RNA isolation by acid guanidine thiocyanate-phenol-chloroform extraction (AGPC method). Anal Biocbem 162, 156-159.

Dimler, R. J., Schaefer, W. C., Wise, C. S. \& Rist, C. E. (1952). Quantitative paper chromatography of D-glucose and its oligosaccharides. Anal Chem 24, 1411-1414.

Dische, Z. (1962). Color reactions of hexosamines. In Methods in Carbobydrate Chemistry, vol. 1, pp. 507-512. Edited by R. L. Whistler \& M. L. Wolfrom. New York: Academic Press.

Gold, S. E. \& Kronstad, J. W. (1994). Disruption of two genes for chitin synthase in the phytopathogenic fungus Ustilago maydis. Mol Microbiol 11, 897-902.

Gow, N. A. R., Swoboda, R., Bertram, G., Gooday, G. W. \& Brown A. J. (1993). Key genes in the regulation of dimorphism of Candida albicans. In Dimorphic Fungi in Biology and Medicine, pp. 61-71. Edited by H. Vanden Bossche, F. C. Odds \& D. Kerridge. New York: Plenum Press.

Holliday, R. (1974). Ustilago maydis. In Handbook of Genetics, vol. 1, pp. 575-595. Edited by R. C. King. New York: Plenum Press.

Maniatis, T., Fritsch, E. F. \& Sambrook, J. (1982). Molecular Cloning: a Laboratory Manual. Cold Spring Harbor, NY: Cold Spring Harbor Laboratory.

Pammer, M. and others (1992). DIT101 (CSD2, CAL1), a cell cycle-regulated yeast gene required for chitin in cell walls and chitosan in spore walls. Yeast 9, 1089-1099.

Ruiz-Herrera, J. (1984). The role of chitosomes in the apical growth of fungi. In Microbial Cell Wall Synthesis and Assembly, pp. 113-120. Edited by C. Nombela. Amsterdam: Elsevier.

Ruiz-Herrera, J. (1992). Fungal Cell Wall: Structure, Synthesis and Assembly. Boca Raton, FL: CRC Press.

Ruiz-Herrera, J. \& Bartnicki-Garcia, S. (1976). Proteolytic activation and inactivation of chitin synthetase from Mucor rouxii. $J \mathrm{Gen}$ Microbiol 97, 241-249.

Ruiz-Herrera, J. \& Xoconostle-Cázares, B. (1995). Molecular and genetic control of chitin biosynthesis in fungi. Arch Med Res 26, 315-321.

Ruiz-Herrera, J., León, C. G., Guevara-Olvera, L. \& Cárabez-Trejo, A. (1995). Yeast-mycelial dimorphism of haploid and diploid strains of Ustilago maydis. Microbiology 141, 695-703.

Sanchez, M., Iglesias, F. J., Santamaria, C. \& Dominguez, A. (1993). Transformation of Kluyveromyces lactis by electroporation. Appl Environ Microbiol 59, 2087-2092.

Sanger, F., Nicklen, S. \& Coulson, A. R. (1977). DNA sequencing with chain-terminating inhibitors. Proc Natl Acad Sci USA 74, 5463-5467.

Shaw, J. A., Mol, P. C., Bowers, B., Silverman, S. J., Valdivieso, M. H., Duran, A. \& Cabib, B. (1991). The function of chitin synthases 2 and 3 in the Saccharomyces cerevisiae cell cycle. J Cell Biol 114,111-123.

Silverman, S. J., Sburlati, A., Slater, M. L. \& Cabib, E. (1988). Chitin synthase 2 is essential for septum formation and cell division in Saccharomyces cerevisiae. Proc Natl Acad Sci US A 85, 4735-4739.

Sudoh, M., Nagahashi, S., Doi, M., Ohta, A., Takagi, M. \& Arisawa, M. (1993). Cloning of the chitin synthase 3 gene from Candida albicans and its expression during yeast-hyphal transition. Mol \& Gen Genet 241, 351-358.

Tsukuda, T., Carleton, S., Fotheringhan, S. \& Holloman, W. K. (1988). Cloning and disruption of Ustilago maydis genes. Mol Cell Biol 8, 3703-3709.

Valdivieso, M. H., Mol, P. C., Shaw, J. A., Cabib, E. \& Duran, A. 
(1991). $C A L 1$, a gene required for activity of chitin synthase 3 in Saccharomyces cerevisiae. J Cell Biol 114, 101-109.

Yanai, K., Kojima, N., Takaya, N., Horiuchi, H., Ohta, A. \& Takagi, M. (1994). Isolation and characterization of two chitin synthase genes from Aspergillus nidulans. Biosci Biotech Biochem 58, 1828-1835.

Yarden, O. \& Yanofsky, C. (1991). Chitin synthase I plays a major role in cell wall biogenesis of Neurospora crassa. Genes $\&$ Dev 5 , $2420-2430$.

Received 20 June 1995; revised 24 August 1995; accepted 16 October 1995. 